\title{
VLBI Monitoring of the most compact sources in M82
}

\author{
D. Fenech ${ }^{* a}$, R. J. Beswick ${ }^{b}$, T. W. B. Muxlow ${ }^{b}$, A. Pedlar ${ }^{b}$ and M. K. Argo ${ }^{c}$ \\ ${ }^{a}$ Department of Physics and Astronomy, University College London, United Kingdom \\ ${ }^{b}$ Jodrell Bank Centre for Astrophysics, University of Manchester, United Kingdom \\ ${ }^{c}$ Netherlands Institute for Radio Astronomy (ASTRON), The Netherlands \\ E-mail: dmfestar.ucl.ac.uk
}

An ongoing investigation of the compact objects within the central kpc of M82, has revealed a number of unusual radio sources. We present the most recent global VLBI observations at 1.7 and $5 \mathrm{GHz}$, which have been used to image these sources with high angular resolutions (4 mas and 1 mas respectively). Previous global VLBI observations at $1.7 \mathrm{GHz}$ from 2005 have also been combined with archival VLBI and EVN observations to monitor the expansion of the supernova remnant $43.31+59.2$ as well as the evolution of the unusual object $41.95+57.5$. We also present some tentative results of the new transient source $43.78+59.3$.

11 th European VLBI Network Symposium \& Users Meeting,

October 9-12, 2012

Bordeaux, France

${ }^{*}$ Speaker. 


\section{Introduction}

M82 is one of the closest examples of a starburst galaxy and is considered a prototypical example. As such, it offers a unique opportunity to study the population of sources associated with a high star-formation rate environment including supernovae and supernova remnants (SNR).

Radio studies of starburst galaxies are essential to performing detailed studies of these populations as they are unobservable at other wavelengths because of the high levels of gas and dust associated with the intense star-formation. As a by-product of the star-formation process, these supernovae and SNR play an important role in the feedback of energy and material into the surrounding interstellar medium.

The proximity of M82 enables the study of a population of young compact radio sources at essentially equivalent angular resolution and brightness sensitivity. In contrast, study of Galactic SNRs is hindered by inaccurate distance estimates and by the fact that the youngest known SNR are over $150 \mathrm{yr}$ old [1].

M82 has become well studied at radio wavelengths and the central kpc of M82 is now known to contain $\sim 100$ discrete sources believed to be predominantly SNR and HII regions. There is also a new supernova SN2008iz [2] and a new radio transient, first detected in May 2009 [3]. The SNR form a population of sources younger and brighter than their Galactic counterparts. As a consequence, since the first observations in the 1960/70s [4, 5], there have been ongoing radio studies, resolving all of the observed sources with either MERLIN or VLBI observations $[6,7,8$, $9,10,11]$.

\section{Global VLBI observations}

A continued high-resolution monitoring programme has been used to study the evolution of two of the most compact sources in M82 since 1986. The observations from this programme have been combined with subsequent observations from 1997 utilising the EVN at $1.7 \mathrm{GHz}$ [8] as well as more recent global VLBI observations made at the same frequency in 1998, 2001, 2005 and 2009 [9, 10, 12]. All such data now form a well established campaign to image and monitor the expansion of a young rapidly evolving supernova remnant, $43.31+59.2$ (see section 2.2) as well as the unusual bi-polar source $41.95+57.5$ (see section 2.1).

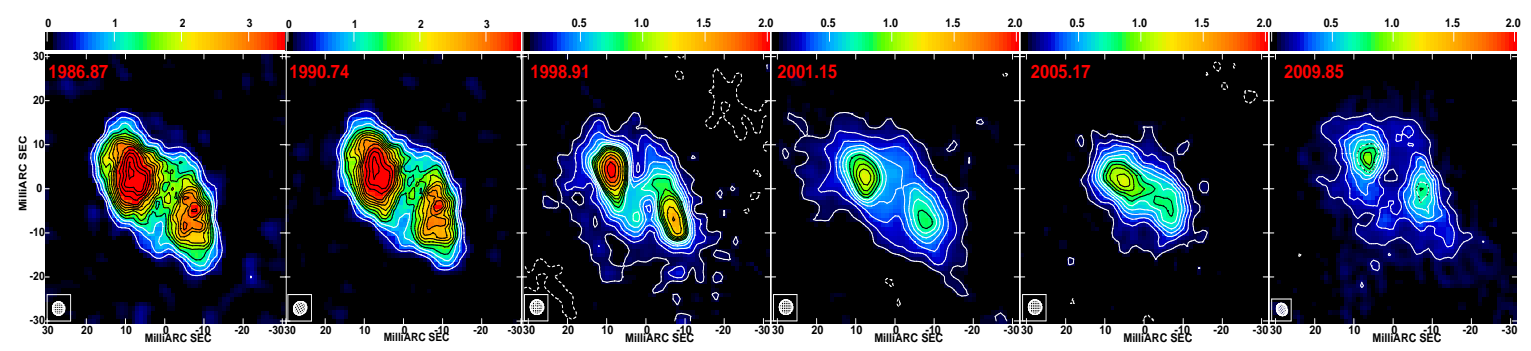

Figure 1: Contour and colour-scale images of $41.95+57.5$ at $1.7 \mathrm{GHz}$ restored with a 3.3 mas circular beam. Contours are plotted at $-1,1,2,3,4,5,6,7,8,9,10 \times 0.31 \mathrm{mJy}_{\text {beam }}^{-1}$ for the first two epochs and $\times 0.11 \mathrm{mJy} \mathrm{beam}^{-1}$ for the latter four. The colour-scale is linear ranging from 0 to $3.5 \mathrm{mJy} \mathrm{beam}^{-1}$ for the first two epochs and from 0 to $2.0 \mathrm{mJy}$ beam $^{-1}$ for the remaining four epochs. 
New $1.7 \mathrm{GHz}$ global VLBI observations were performed in July 2012 using a twenty element array. This incorporated eleven of the EVN antennas and nine of the ten VLBA antennas. These observations will provide the most high-fidelity, high-resolution images of the compact sources within M82. Observations of the phase reference source J0958+65 were made by switching between this and M82. The sources 3C84 and J0927+39 were also observed and used in the fringe-fitting and bandpass calibration.

\section{1 $41.95+57.5$}

Until the appearance of the new supernova, SN2008iz and the transient source 43.78+59.3, $41.95+57.5$ was the most compact source within M82. This source has been characterised by its distinct bi-polar structure, atypical of the ring-like morphology expected of SNR. Early observations of $41.95+57.5$ at $2.3 \mathrm{GHz}$ and $5 \mathrm{GHz}$ showed a structure indicative of an elongated shell, hence its initial classification as a SNR $[13,14]$. Further observations at $1.7 \mathrm{GHz}$ using the EVN have confirmed this elongated structure $[8,10]$.

The 15 mas beam of the EVN-only observations does not fully resolve this source and, as a result, only the global VLBI observations have been used to monitor its evolution on scales of a few mas, corresponding to $\sim 0.05 \mathrm{pc}$ [10]. However, archival global VLBI observations from 1987 and 1990 have now been combined with the later global epochs [14, 15]. Fig. 1 shows contour and colour-scale images of this source from all available global VLBI observations at $1.7 \mathrm{GHz}$ up until 2009.

The global VLBI observations performed in 2009 and 2012 included both $1.7 \mathrm{GHz}$ and $5 \mathrm{GHz}$ data. Fig. 2 shows the $20095 \mathrm{GHz}$ image (centre) as well as images at both observed frequencies from 2012. This figure clearly shows that there may in fact be more extended emission associated with this source, as a ring-like structure can be seen at both frequencies and both epochs as well as the previously observed bi-polar emission.
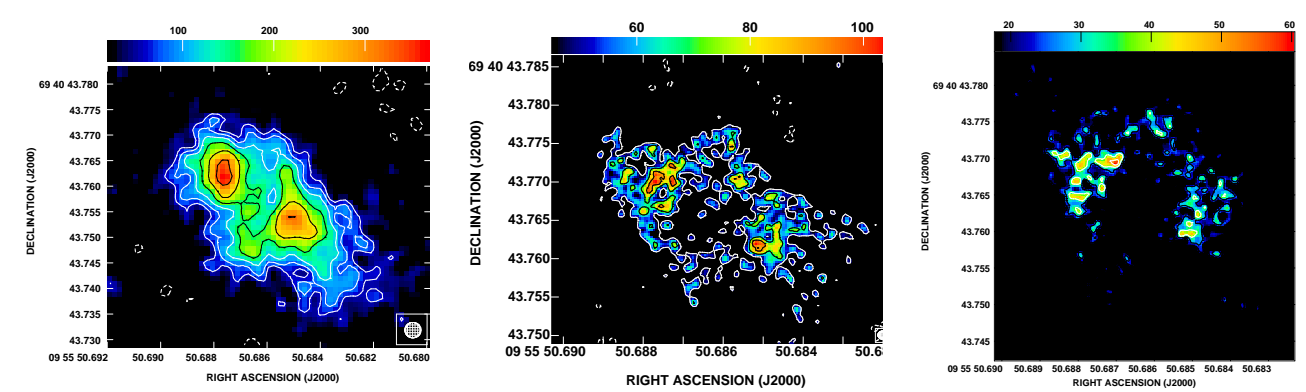

Figure 2: Contour and colour-scale images of 41.95+57.5. Left: $20121.7 \mathrm{GHz}$ image restored with a 3 mas circular beam. Contours are plotted at $-1,1,2,3,4,5,6,7,8,9,10 \times 0.11 \mathrm{mJy}^{-1}$ beam $^{-1}$ and the colour-scale is linear ranging from 0 to $400 \mu \mathrm{Jy} \mathrm{beam}^{-1}$. Centre and right: 2009 and 2012 (respectively) $5 \mathrm{GHz}$ images restored with a 1 mas circular beam. Contours are plotted at $-1,1,1.5,2, \ldots, 4.5,5 \times 45 \mu \mathrm{Jy} \mathrm{beam}^{-1}$. The colour-scale is linear ranging from 45 to $105 \mu \mathrm{Jy}_{\text {beam }}{ }^{-1}$ (centre) and from 18 to $62 \mu \mathrm{Jy}_{\text {beam }}{ }^{-1}$ (right).

$41.95+57.5$ is also unique in that it continues to display a decay in flux density at a rate of $\sim 8.5 \%$ per year. This decay has been evident from the first observations of M82 in the early 1960 's.

Muxlow et al. 2005 [16] suggested that $41.95+57.5$ could be an evolved gamma-ray burst (GRB) remnant, citing that the bi-polar structure could be explained by the oppositely directed 

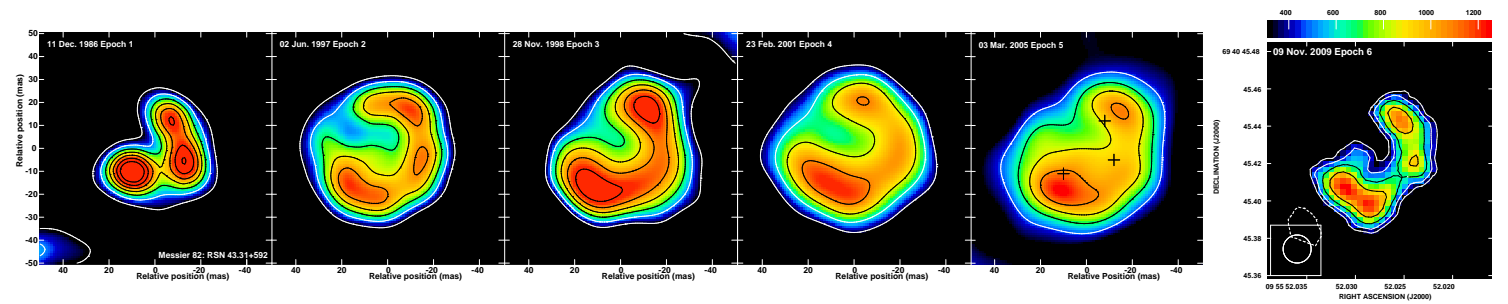

Figure 3: Contour and colour images of the SNR 43.31+59.2 at 1.7 GHz from each VLBI epoch. Contours are plotted at $-1,1,2,3,4,5,6,7,8,9,10 \times 0.35 \mathrm{mJy}$ beam $^{-1}$ for all epochs. The colour-scale is linear ranging from 0.1 to $2.2 \mathrm{mJy}_{\mathrm{beam}} \mathrm{b}^{-1}$ for the first three epochs, from 0.1 to $1.7 \mathrm{mJy} \mathrm{beam}^{-1}$ for the remaining two in the left image (taken from [12]) and from 0.32 to $1.25 \mathrm{mJy} \mathrm{beam}^{-1}$ for the 2009 epoch (far right).

jets associated with a GRB event. The tentative emerging ring-like structure associated with this source could give support to the idea that this source originated as an unusual and bright supernova event such as a GRB. There is however, continued speculation as to the true nature of this source. The unusual supernova SN1986J in NGC891 also showed characteristics atypical of the expected expanding-shell scenario, and similar to those of 41.95+57.5, with an elongated morphology [17]. VLBI observations of SN1986J in 2004 revealed the emergence of a compact core believed to be a pulsar or an accreting black hole [18]. The higher resolution global VLBI observations from 2009 and 2012 at $5 \mathrm{GHz}$ provide no obvious sign of a compact core developing which could be expected if the nature of $41.95+57.5$ were to prove a source of similar origin to that of SN1986J.

\section{$2.243 .31+59.2$}

In contrast to the unusual nature of $41.95+57.5,43.31+59.2$ shows a very well-defined shell structure (see Fig. 3). In addition, this source, believed to be the youngest of the SNR within M82, appears to be undergoing a more typical evolution. Investigations using previous VLBI observations (e.g. $[8,9,10]$ ) have shown $43.31+59.2$ to be expanding at $\sim 10^{4} \mathrm{~km} \mathrm{~s}^{-1}$. More recently, a comparison of the 2005 global VLBI observations with previous epochs yielded a slightly lower expansion velocity of $7600 \pm 1800 \mathrm{~km} \mathrm{~s}^{-1}$ [12]. This could indicate that the expansion has begun to decelerate and a comparison of velocities determined from various epochs shows tentative evidence for such a trend (see Fig. 8 in [12])

The evolution of a SNR can be parametrised as $D=k T^{\delta}$, where $D$ is the size of the remnant and $T$ is its age. $\delta$ represents the deceleration parameter and $k$ is a constant [9]. Incorporating the later epochs of global VLBI observations into this analysis should provide sufficient data to determine the deceleration parameter for $43.31+59.2$, which has previously only been defined as a lower limit of $0.53 \pm 0.06$ (for a full discussion see [12]). This will provide important information as to how this SNR evolves and what the effect the surrounding environment will have on its evolution.

\subsection{The not-so transient source, $43.78+59.3$}

A new source, 43.78+59.3, was detected in MERLIN observations in May 2009 [3]. This source was initially considered to be one of the population of transient sources to appear in M82. However, following its initial detection, $43.78+59.3$ increased in flux density to $\sim 700 \mu \mathrm{Jy} \mathrm{beam}^{-1}$ within approximately eight days and has remained at this level in subsequent observations [3]. This 
source is now being regularly monitored using MERLIN/e-MERLIN, eVLBI and global VLBI observations to determine its nature.

A new radio supernovae is expected to undergo a rapid rise followed by a power-law decline with a progressive turn-on with frequency. However, 43.78+59.3 experienced a rapid rise in flux density to a constant level and appeared to have a steep spectral index at turn-on, which has also shown virtually no variation since.

As a result this source does not appear to be a typical radio supernovae and the fundamental nature of this transient source remains unknown. Muxlow et al. (2010) discussed several possibilities that could provide some explanation. These included a peculiar supernova event, a faint active galactic nucleus (AGN) at the centre of the galaxy as well as a microquasar [3]. However, none of these suggestions currently fully explain the observed characteristics.

Muxlow et al. (2010) also reported a tentative detection of superluminal motion of $\sim 4.2 \mathrm{c}$ within the MERLIN monitoring observations of this source [3]. This motion appeared to be in an east-west direction. As can be seen from the global VLBI images of the transient source in Fig. 4, the $5 \mathrm{GHz}$ image shows a clear elongated structure in the east-west direction, consistent with the motion reported in [3].
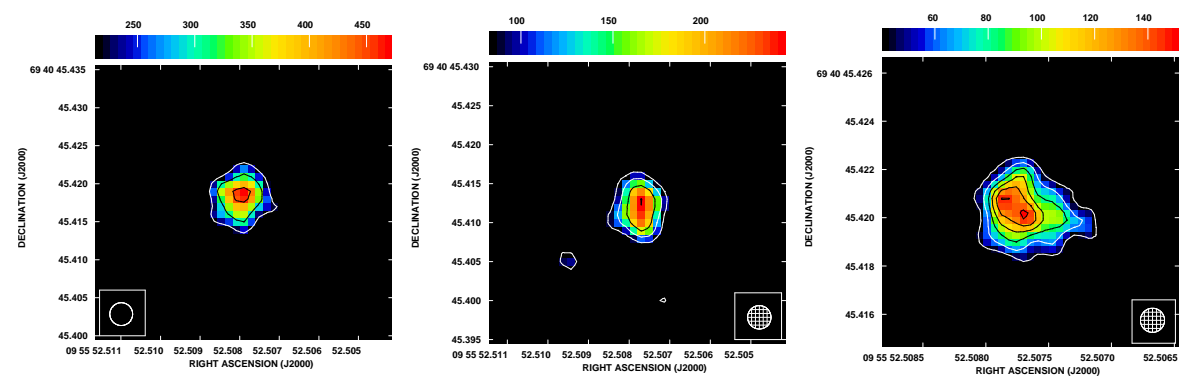

Figure 4: Contour and colour-scale images of 43.78+59.3 from global VLBI observations at $1.7 \mathrm{GHz}$ in 2009 (left) and 2012 (centre) and at $5 \mathrm{GHz}$ in 2009 (right). The contours are plotted at $-1,1,1.5,2, \ldots 5 \times 0.042 \mathrm{mJy}_{\text {beam }}^{-1}$ for the $1.7 \mathrm{GHz}$ epochs and $-1,1,1.4,2,2.8,4,5.7,8,11.3,16 \times 0.085 \mathrm{mJy}_{\text {beam }}^{-1}$ for the $5 \mathrm{GHz}$ epoch.

\subsection{VLBI compact sources}

Five compact sources were detected in the early EVN observations of M82 from 1986. However, only $41.95+57.5$ and $43.31+59.2$ have been extensively studied. Of the remaining three sources, $43.18+58.2$ became too faint and resolved beyond the first epochs, to be detected in subsequent observations. The remaining two sources, 44.01+59.6 and 45.17+61.2, have been repeatedly studied with MERLIN and are relatively compact at the available $\sim 50$ mas resolution. Both 44.01+59.6 and 45.17+61.2 were detected in the EVN observations from 1997. However, the higher noise level of the subsequent epoch made it difficult for accurate assessment of their evolution. Fig. 5 shows the source from the EVN and subsequent global VLBI epochs. Given the addition of the more recent wide-field global VLBI observations, it should now be possible to investigate the expansion of these sources along with that of $41.95+57.5$ and $43.31+59.2$. This will include confirming the expansion velocities determined using MERLIN observations for these sources [11]. 

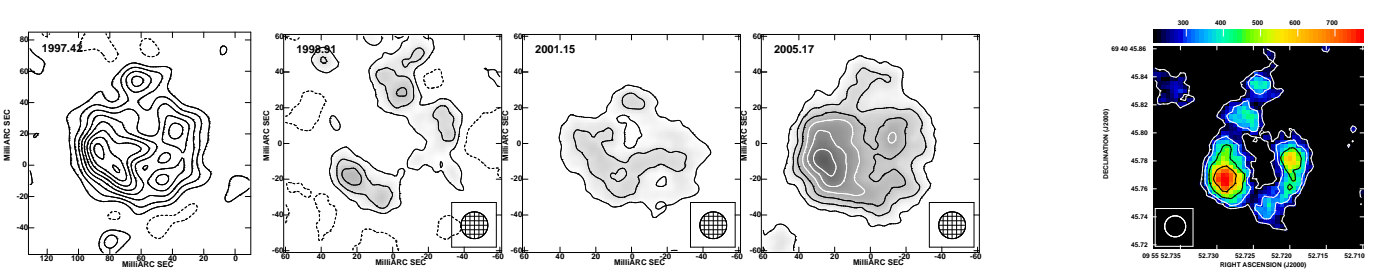

Figure 5: Left: Gray-scale and contour images of $44.01+59.6$ at 15 mas resolution from four $1.7 \mathrm{GHz}$ global VLBI epochs between 1997 and 2005. Contours are plotted at $-1,1,2, \ldots 10 \times 0.1 \mathrm{mJy}_{\text {beam }}{ }^{-1}$ in all epochs (taken from [12]). The grey-scale ranges from $3 \sigma$ to $1.0 \mathrm{mJy} \mathrm{beam}^{-1}$ where $\sigma$ is 0.005 in 1998, 0.003 in 2001 and $0.004 \mathrm{mJy} \mathrm{beam}^{-1}$ in 2005 (no grey-scale is plotted in the 1997 epoch). Right: Contour and colour-scale image of $44.01+59.2$ at 15 mas resolution from an additional $1.7 \mathrm{GHz}$ global VLBI epoch in 2009. Contours are plotted at $-1,1,1.4,2,2.8,4,5.7,8$, $11.31,16 \times 0.22 \mathrm{mJy} \mathrm{beam}^{-1}$. The colour-scale ranges from 0.22 to $0.77 \mathrm{mJy} \mathrm{beam}^{-1}$.

\section{Summary}

The latest epoch of global VLBI observations of M82 has been used to image the unusual source 41.95+57.5. Alongside the previous archival global VLBI data, this has shown the appearance of a ring-like component. In addition, the evolution of the SNR 43.31+59.2 has been further studied through comparison of the previous VLBI observations. It is hoped the most recent observations will begin to constrain the nature of the expansion of this source and determine any deceleration. The transient source, $43.78+59.3$, has also been detected in these recent global VLBI observations with preliminary images at $5 \mathrm{GHz}$ showing an elongated structure along the same axis as the tentative superluminal motion reported by [3].

\section{References}

[1] D. Green, MNRAS 387 (2008) 54

[2] A. Brunthaler et al., $A \& A \mathbf{5 1 6}$ (2010) 27

[3] T.W.B. Muxlow et al., MNRAS 404 (2010) 109

[4] E. M. Burbridge, G. R. Burbridge, V. C. Rubin, ApJ 140 (1964) 942

[5] P. P. Kronberg, P. N. Wilkinson, ApJ 300 (1975) 430

[6] S.W. Unger, A. Pedlar, D.J. Axon, P.N.Wilkinson, P.N. Appleton, MNRAS 211 (1984) 783

[7] P. P. Kronberg, P. Biermann, F. R. Schwab, ApJ 291 (1985) 693

[8] A. Pedlar, MNRAS 307 (1999) 761

[9] A.R. McDonald et al., MNRAS 322 (2001) 100

[10] R.J. Beswick et al., MNRAS 369 (2006) 1221

[11] D.M. Fenech, T.W.B. Muxlow, R.J. Beswick, A. Pedlar and M.K. Argo, MNRAS 391 (2008) 1384

[12] D.Fenech, R.J. Beswick, T.W.B. Muxlow, A. Pedlar and M.K. Argo, MNRAS 408 (2010) 607

[13] N. Bartel et al., ApJ 323 (1987) 505

[14] P.N. Wilkinson, A.G. deBruyn, MNRAS 242 (1990) 529

[15] W.M. Trotman, MSc Thesis, University of Manchester (1996)

[16] T.W.B. Muxlow et al., Memorie S. A. It 76 (2005) 586

[17] M. F. Bietenholz, N. Bartel, M.P. Rupen, ApJ 581 (2002) 1132

[18] M. F. Bietenholz, N. Bartel, M.P. Rupen, Science 304 (2004) 1947 